\title{
PHYSICAL PROPERTIES OF THE INTERPLANETARY DUST
}

\author{
MARTHA S. HANNER \\ Dudley Observatory
}

The interplanetary dust may be composed of cometary material, interstellar grains, debris from asteroidal collisions, primordial material formed by direct condensation, or contributions from all of these sources. Before we can determine the origin of the dust, we need to know its physical nature, spatial distribution, and the dynamical forces that act on the particles. The spatial distribution and dynamics are separately treated in this symposium by Roosen. ${ }^{1}$ We discuss here the physical characteristics of the dust particles: their size distribution, chemical composition, physical structure, and optical properties.

Data on these properties can be obtained from particle collections, impact craters, thin-film penetrations, acoustic detectors, and the zodiacal light. The results obtained from various methods have been reviewed by Singer and Bandermann (1967) and Bandermann (1968). We shall concentrate on discussing the information that can be derived from observations of the zodiacal light.

\section{ZODIACAL LIGHT MODELS}

The zodiacal light provides information on the average properties of the interplanetary dust over a large volume of space. Analysis of the brightness and polarization of the zodiacal light yields, in principle, the large-scale size distribution, spatial distribution, and optical properties of the scattering particles. The optical properties, in turn, contain information on the composition and physical structure of the dust particles.

Figure 1 illustrates the basic scattering geometry. Particles in the volume element at $P$, at a distance $r$ from the Sun, scatter sunlight through an angle $\theta$ into the line of sight along $P E$. The contribution to the observed brightness from all the particles in the volume element at $P$ will be

$$
d I_{\lambda}(\epsilon)=\frac{E_{0} R_{0}^{2}}{r^{2}} d \Delta \int_{a_{1}}^{a_{2}} n(a, r) F_{\lambda}(a, \theta) d a
$$

${ }^{1}$ See p. 363. 


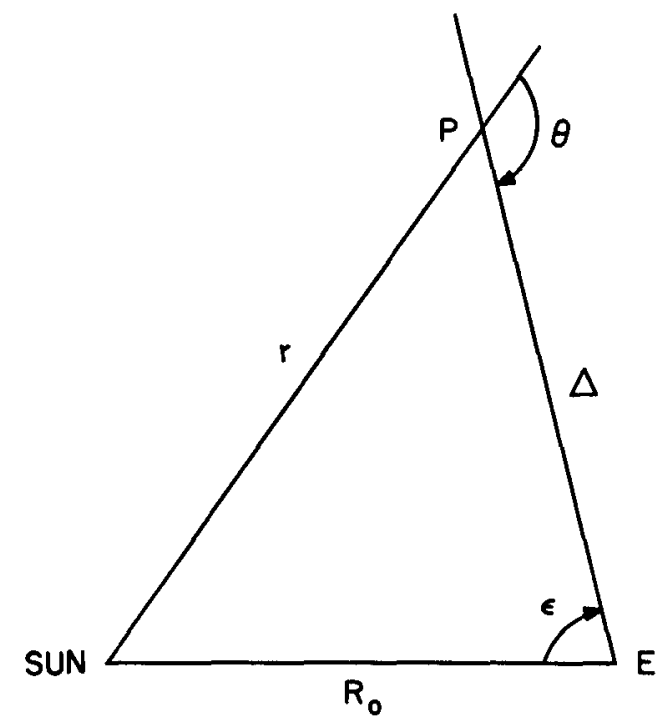

Figure 1.-Basic scattering geometry for the zodiacal light.

where

$$
\begin{aligned}
E_{0} & =\text { solar flux at } 1 \mathrm{AU} \\
R_{0} & =1 \mathrm{AU}
\end{aligned}
$$

$n(a, r)=$ particle size and spatial distribution

$a_{1}, a_{2}=$ the lower and upper limits to the particle size distribution

$F_{\lambda}(a, \theta)=$ angular scattering function for particles of radius $a$

The total surface brightness at an elongation $\epsilon$ from the Sun will be the integral of $d I(\epsilon)$ over the line of sight

$$
I_{\lambda}(\epsilon)=E_{0} R_{0} \int_{\Delta=0}^{\infty} \int_{a_{1}}^{a_{2}} \frac{n(a, r)}{r^{2}} F_{\lambda}(a, \theta) d a d \Delta
$$

In practice, there is no simple way of separating the parameters in equation (2). The size distribution can be expected to be a function of $r$ because the dynamical forces acting on the dust depend on particle dimension as well as density. The scattering functions themselves may vary with distance from the Sun because solar radiation can alter the optical properties of the dust particles. The interplanetary material is probably a mixture of several components having different relative concentrations in different parts of the solar system. Cometary and asteroidal debris, for example, would not be injected at the same solar distance. 
The approach that has generally been applied has been to choose a form for $F_{\lambda}(a, \theta)$, assume that the particle size distribution is independent of the spatial distribution, and adopt a functional form for each that is based either on convenience, on the results of dynamical calculations, or on other observational evidence, such as data from acoustic and penetration experiments or extrapolation of meteor fluxes. Models of the brightness and polarization of the zodiacal light are then computed and the model parameters adjusted until a fit with the observations is obtained. Even if a good fit is achieved, there is, of course, no guarantee that the model so obtained is unique, and a variety of models have been found to represent the data about equally well. Reviews of theoretical work have been given by Blackwell, Dewhirst, and Ingham (1967), Elsässer (1963), and others.

The earliest models, which assumed an isotropic scattering function, made it necessary to postulate a high particle concentration near the Sun to account for the steep increase in brightness in the $F$ corona. Allen (1946) and van de Hulst (1947) independently showed that the brightness distribution in the $F$ corona can be accounted for by diffraction without having to assume large numbers of dust particles close to the Sun. Ingham (1961) and Blackwell, Ingham, and Petford (1967) also treated the scattering function as a diffraction component plus a reflection component in deriving the albedo and the spatial distribution from coronal observations. Separating the scattering function into reflection and diffraction components is valid at all scattering angles for particles much larger than the wavelength. At small scattering angles, the diffraction component will be adequate for spherical particles a few micrometers in size at optical wavelengths. Diffraction will predict too steep a rise in intensity in the forward direction for particles less than $1 \mu \mathrm{m}$ in size. (See van de Hulst, 1957, and Giese, 1961.)

With the introduction of high-speed computers, many authors have generated models using Mie theory to compute the scattering functions. The most extensive calculations have been carried out by Giese (1961), Giese and Siedentopf (1962), Giese (1963), and Giese and Dziembowski (1967). Similar studies have been done by Little, O'Mara, and Aller (1965); Elsässer and Schmidt (1966); Aller et al. (1967); Powell et al. (1967); and Hanner (1970).

\section{COMPARISON OF THEORY AND OBSERVATION}

Most of the observations available for comparison with theoretical models are restricted to the ecliptic plane. Even here there are wide discrepancies among the results of different observers, due in part to the difficulties in correcting for atmospheric scattering and in separating the zodiacal light from the airglow and integrated starlight. These problems have been discussed by Weinberg (1970). The most extensive published data on the distribution of the zodiacal light brightness away from the ecliptic are by Smith, Roach, and Owen (1965). Their observations were made at only one wavelength, so that models that fit their data at $530 \mathrm{~nm}$ cannot be tested over a wide wavelength 
range. Ingham and Jameson (1968) and Jameson (1970) observed the brightness and polarization at $510 \mathrm{~nm}$ in regions away from the ecliptic.

Model calculations indicate that the polarization is a more sensitive discriminant than the intensity in determining the optical properties and size distribution of the dust particles. The maximum observed polarization $P_{\mathrm{max}}$ in the ecliptic (about 23 percent at $530 \mathrm{~nm}$ ) occurs near $\epsilon=70^{\circ}$ (Weinberg, 1964). For a reasonably smooth spatial distribution, regardless of the exact form, a maximum near $\epsilon=70^{\circ}$ implies a maximum polarization at scattering angles near $90^{\circ}$. On the basis of Mie theory, the maximum polarization occurs near $\theta=90^{\circ}$ (and thus $\epsilon=70^{\circ}$ ) only if a significant number of particles have radii on the order of $0.1 \mu \mathrm{m}$ or less. This is true for both dielectric and metallic particles. For $n(a) \propto a^{-p}$ this means $p \geqslant 4$ and $a_{1} \lesssim 0.1 \mu \mathrm{m}$. When the particle size distribution is weighted toward larger radii, Mie theory predicts the following changes:

(1) Metals: $P_{\max }$ shifts to smaller scattering angles $\left(\theta<90^{\circ}\right)$.

(2) Ices $(m \sim 1.3): P_{\max }$ shifts to larger angles $\left(\theta \sim 130^{\circ}\right.$ to $\left.150^{\circ}\right)$.

(3) Silicates $(1.5 \leqslant m \leqslant 1.75)$ : Large amounts of negative polarization occur over a wide range of scattering angles.

Thus, a fit to the observed polarization in the ecliptic plane with a single-component model requires that the scattering particles be predominantly of radius $a \lesssim 0.1 \mu \mathrm{m}$.

Both Giese and Dziembowski (1967) and Aller et al. (1967) found that the single-component model $(m=1.3)$ giving the best fit to the intensity and polarization in the ecliptic did not match the data of Smith, Roach, and Owen away from the ecliptic. Giese and Dziembowski proposed a two-component model consisting of silicates and iron particles, $n(a) \propto a^{-2.5}$. By adjusting the cutoff sizes and the relative concentration of the two components, they were able to produce a polarization maximum near $70^{\circ}$ and to improve the fit to the observed brightness perpendicular to the ecliptic.

The presence of negative polarization near the antisolar direction has been discussed by Weinberg and Mann (1968). They find that the polarization reversal occurs near $\epsilon=165^{\circ}$ at $508 \mathrm{~nm}$ and shifts toward smaller elongation at longer wavelength. Mie theory predicts negative polarization at large scattering angles for dielectric particles less than $1 \mu \mathrm{m}$ in size consisting of either ices or silicates. The negative polarization extends over a wider range of scattering angles at larger refractive index. For refractive index $m=1.3$, the appropriate choice of size and spatial distribution can produce a neutral point near $\epsilon=165^{\circ}$ at $508 \mathrm{~nm}$. However, for a power-law size distribution, the position of the neutral point shifts to larger $\epsilon$ at longer wavelength.

The published observational data have limited coverage in both space and time as well as a limited range in wavelength. In spite of the amount of effort that has been expended in analyzing these observations, very few definitive conclusions can be drawn regarding the physical nature of the dust particles. On the theoretical side, too much emphasis has been placed on adjusting 
mathematical parameters to fit a specific idealized model to a limited set of observations. We must take a broader approach and ask what qualitative features can be used to discriminate between different kinds of scattering particles and what observational data will be most valuable for this purpose.

The wavelength dependence of polarization is a critical parameter in determining the size range and physical nature of the dust particles. More extensive data are needed from a good ground-based site, covering the entire sky over a wide range of wavelengths.

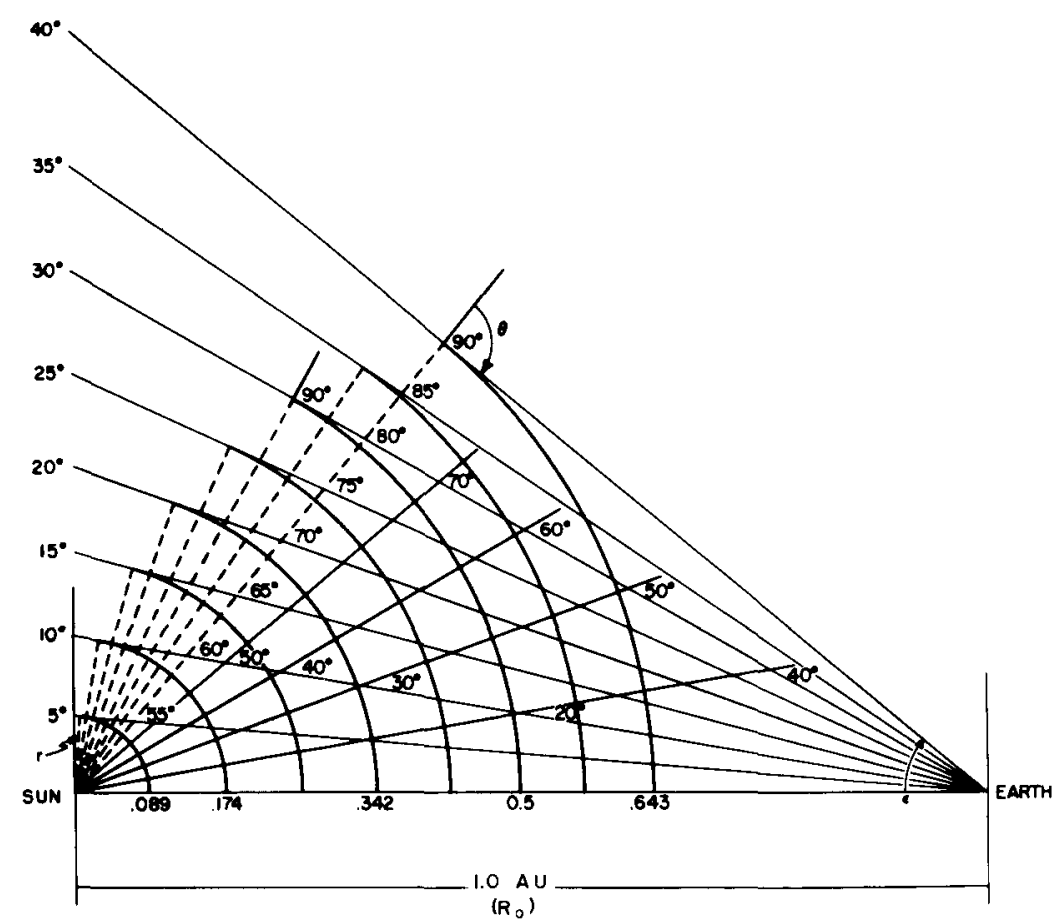

Figure 2.-Variation of scattering angle and solar distance along line of sight as a function of elongation (J. L. Weinberg, personal communication).

Ground-based observations have several limitations, however. Corrections for tropospheric scattering and airglow emission are uncertain. The ultraviolet and infrared spectral regions cannot be observed. Observations outside of eclipse are restricted to $\epsilon \geqslant 30^{\circ}$. Figure 2 shows the region of space sampled by the line of sight for a series of elongation angles. It can be seen that at $\epsilon=30^{\circ}$ we can get no information about the region closer than $0.5 \mathrm{AU}$ to the Sun. It is therefore important to obtain detailed observations from satellites to supplement the ground-based data. The following types of observations are of particular value. 


\section{Observations in the Ultraviolet}

The scattering properties of small particles depend on the quantity $2 \pi a / \lambda$. Thus in the ultraviolet the particles "look" larger, and a better discrimination between different size distributions will be possible. We would expect the $I_{\lambda}(\epsilon)$ curve to have a steeper slope in the ultraviolet at $\epsilon<90^{\circ}$ and the position of maximum in the $P_{\lambda}(\epsilon)$ curve to shift. Assuming Mie theory provides at least a qualitative indication of the particle scattering properties, we expect the maximum polarization to shift toward larger $\epsilon$ if the particles are dielectric (ices or silicates) and toward smaller $\epsilon$ if the particles are metallic. The two-component model of Giese and Dziembowski would show a broadened maximum or perhaps a double maximum. Negative polarization may appear either at small angles or near the backscatter direction depending on the particle sizes and refractive index. The larger $a / \lambda$ also means that effects of shape and surface irregularities will show up more strongly in the ultraviolet.

More fundamentally, many materials have a change in their optical properties in the ultraviolet region (Taft and Philipp, 1965; Field, Partridge, and Sobel, 1967). Thus it is important to search for "signatures" of certain materials by studying the wavelength variation of the intensity and polarization in the ultraviolet. The interstellar extinction curve, for example, shows a bump near $220 \mathrm{~nm}$ (Stecher, 1965). The presence of unusual features in the zodiacal light at $220 \mathrm{~nm}$ could have interesting implications in relating the interplanetary and interstellar grains.

\section{Observations at Small Elongations}

The importance of relating observations of the $F$ corona and the zodiacal light has been stressed by van de Hulst (1947), Blackwell and Ingham (1967), and many others. Observations of the $F$ corona made during eclipse are difficult to relate to zodiacal light data, for almost no data exist in the intervening region. The region at small $\epsilon$ is critical for several reasons:

(1) The change in slope of $I_{\lambda}(\epsilon)$ with $\lambda\left(5^{\circ} \leqslant \epsilon \lesssim 50^{\circ}\right)$ provides information on the size of the scattering particles.

(2) The amount and direction of polarization, particularly in the ultraviolet, may indicate the nature of the particles because certain materials and certain sizes show negative polarization at small scattering angles.

(3) Comparison of the observed brightness at small $\epsilon$ with theoretical models may give information on the extent of a dust-free zone surrounding the Sun (Elsässer, 1970).

(4) Particles close to the Sun may show a change in their optical properties as a result of radiation damage.

(5) The size distribution may be modified by sputtering and vaporization (Singer and Bandermann, 1967). 


\section{Simultaneous Satellite and Ground-Based Observations}

The large body of ground-based data would become more valuable if accurate methods of correcting for tropospheric scattering and airglow emission can be developed. Simultaneous observations from the ground and from a satellite using similar instruments and wavelength bands can provide direct information on the effects of Earth's atmosphere. Such observations should be carried out over an extended period of time so that effects of variation in airglow emission and possible variations in the zodiacal light can be evaluated.

\section{Infrared Observations}

Observations in the infrared at small $\epsilon$ can provide information on the thermal properties of the dust particles and the extent of the dust-free zone surrounding the Sun (Peterson, 1963, 1964; Harwit, 1963). Eclipse observations at 2.2 and $3.5 \mu \mathrm{m}$ have been obtained by Peterson and MacQueen (1967) that show a maximum at 4.0 and a secondary peak at 3.5 solar radii (distance from the center of the Sun). Satellite data can provide more extensive coverage in space and time.

\section{SCATTERING BY IRREGULAR PARTICLES}

Many of the theoretical models of the zodiacal light brightness and polarization used to draw conclusions concerning the particle size distribution and composition have been based on the scattering functions for smooth, homogeneous, spherical particles, whereas the dust particles gathered in collection experiments are generally irregular, even fluffy in appearance (Hemenway et al., 1967; Hemenway and Hallgren, 1970; Hemenway et al., 1970). The assumption is implicitly made that an extended size distribution of randomly oriented, irregular particles will scatter light in the same manner as the same size distribution of spheres. There are little experimental data available for particles of size $a \sim \lambda$.with which to test the validity of this assumption.

Donn and Powell (1962) have studied the scattering by a size distribution of zinc oxide crystals, $m=2.01$, which grow in the form of spikes. They found that the scattering characteristics for a size distribution of such particles could be represented by a distribution of much smaller spheres with $m=2.01$ or moderately smaller spheres with refractive index as low as 1.2. Their work and other research on irregular particles have been summarized by Powell et al. (1967), who conclude that the scattering by a size distribution of large-volume particles such as cubes can be described by a size distribution of spheres very close to the real size distribution. They find that the angular dependence of the intensity and polarization at different wavelengths can be duplicated by the same distribution of spheres. For small-volume particles such as needles and fourlings, the equivalent size distribution of spheres that matches their data at 
one wavelength will not fit at another wavelength. In addition, the sizes of the spheres will be much smaller than the characteristic lengths of the actual particles.

Holland and Gagne (1970) measured the scattering matrix for a polydisperse system of silica particles smaller than $1 \mu \mathrm{m}, m=1.55$. Their data for the matrix elements $S_{11}$ and $S_{22}$ at $546 \mathrm{~nm}$ are reproduced in figure 3 (Holland, 1969). The solid curve was computed from Mie theory for the observed size distribution. Mie theory fits the data fairly well at small scattering angles, but it predicts a steep rise toward the backscatter direction that is absent in the laboratory data. Data for $S_{12}=S_{21}$ indicate that the polarization is positive at $\theta \lesssim 160^{\circ}$, whereas Mie theory predicts negative polarization. There is, however, an indication that the polarization changes sign near $160^{\circ}$ at $546 \mathrm{~nm}$ and near $150^{\circ}$ at $486 \mathrm{~nm}$. The position of the neutral point varies with wavelength in the opposite direction from the shift observed by Weinberg and Mann in the zodiacal light.

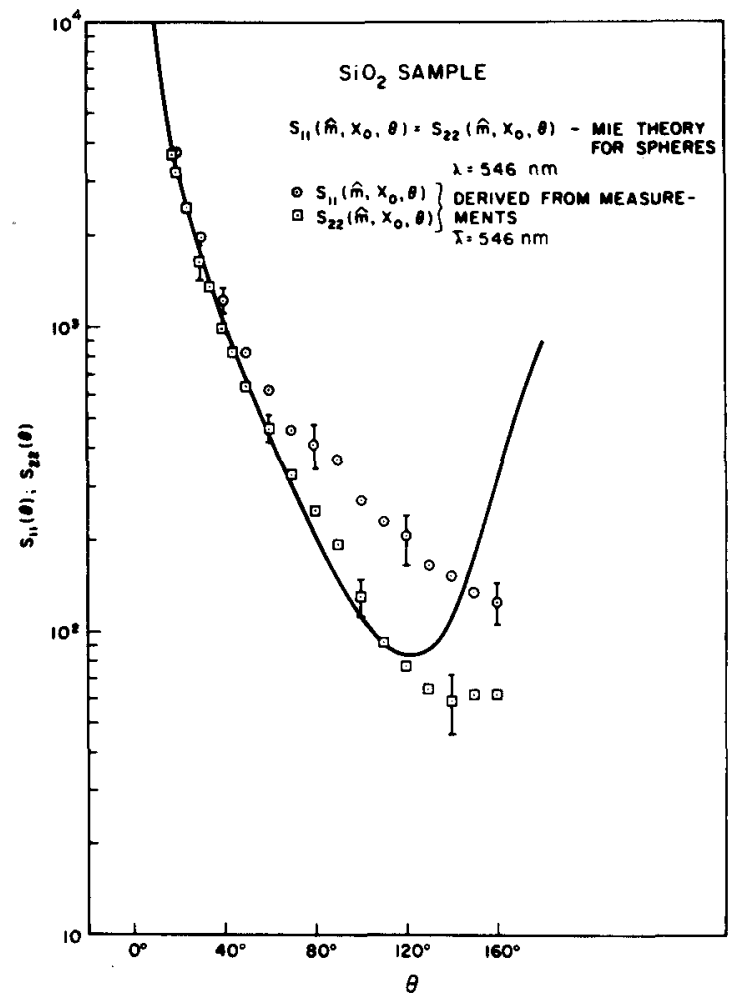

Figure 3.-Variation of matrix elements $S_{11}(\theta)$ and $S_{22}(\theta)$ with scattering angle $\theta$. Solid line is theoretical curve computed from Mie theory (Holland, 1969, fig. 6). $\hat{m}=$ refractive index; $X_{0}=$ size distribution parameter. 
The scattering properties of nonspherical particles also can be studied by microwave scattering from scaled particle models (Greenberg, Pedersen, and Pedersen, 1961). Greenberg, Wang, and Bangs (1971) have found that the measured extinction for particle models with roughened surfaces differs widely from the extinction by smooth spheres. Microwave scattering data over the whole range of scattering angles for many values of $a / \lambda$ are needed before we can conclude whether the peculiar scattering patterns observed for individual particles average out to resemble scattering by spheres over an extended size distribution.

Scattering functions can be computed analytically for the case of long cylinders (Kerker, 1969; Lind, 1966). Detailed comparison of the scattering functions for spheres and cylinders, and possibly ellipsoids, can provide useful information on the effects of particle shape. For example, there is a significant change in the polarization, even for an extended size distribution, when the angle between the cylinder axis and the incident radiation is varied. However, the polarization by randomly oriented cylinders with $n(a) \propto \exp \left[-5(a / 0.5)^{3}\right]$ was quite similar to that for spheres with the same size distribution (Hanner, 1969).

The limitations of Mie theory and the importance of computing zodiacal light models for nonspherical particles have been emphasized by Greenberg (1970). Richter (1966) has discussed experimental phase functions and polarization curves for irregular particles over the size range $10^{-6}$ to $10 \mathrm{~cm}$.

\section{CONCLUSION}

The zodiacal light data sample the average properties of the interplanetary dust particles over a large volume of space. Intensity and polarization measurements in the ultraviolet and infrared, together with the wavelength dependence of polarization throughout the visible spectral region, will provide information on the physical nature and size distribution of the dust particles. However, we cannot expect to obtain a complete model of the interplanetary dust from zodiacal light observations alone. The data will be most valuable when combined with the results of particle collections and other methods used to study in detail the physical properties of individual particles.

Giese and Dziembowski (1969) and Giese (1970) have discussed the value of zodiacal light observations from space probes in determining the spatial distribution of the interplanetary dust. Zodiacal light experiments will be included on both the Helios inner solar system probes and the Pioneer F and G asteroid-Jupiter probes.

\section{ACKNOWLEDGMENTS}

It is a pleasure to thank Dr. J. L. Weinberg for his interest and his helpful discussions. This research has received support from NSF grant GA-12400 and NASA grant NGR 33-017011. 


\section{REFERENCES}

Allen, C. W. 1946, The Spectrum of the Corona at the Eclipse of 1940 October 1. Mon. Notic. Roy. Astron. Soc. 106, 137.

Aller, L. H., Duffner, G., Dworetsky, M., Gudehus, D., Kilston, S., Leckrone, D., Montgomery, J., Oliver, J., and Zimmerman, E. 1967, Some Models of the Zodiacal Cloud. The Zodiacal Light and the Interplanetary Medium (ed., J. L. Weinberg), pp. 243-256. NASA SP-150.

Bandermann, L. W. 1968, Physical Properties and Dynamics of Interplanetary Dust. Ph. D. Thesis. Univ. of Maryland.

Blackwell, D. E., Dewhirst, D. W., and Ingham, M. F. 1967, The Zodiacal Light. Advances in Astronomy and Astrophysics (ed., Z. Kopal), vol. 5, p. 1. Academic Press, Inc. New York.

Blackwell, D. E., and Ingham, M. F. 1967, Toward a Unification of Eclipse and Zodiacal-Light Data. The Zodiacal Light and the Interplanetary Medium (ed., J. L. Weinberg), pp. 17-21. NASA SP-150.

Blackwell, D. E., Ingham, M. F., and Petford, A. D. 1967, The Distribution of Dust in Interplanetary Space. Mon. Notic. Roy. Astron. Soc. 136, 313.

Donn, B., and Powell, R. S. 1962, Angular Scattering From Irregularly Shaped Particles With Application to Astronomy. Electromagnetic Scattering (ed., M. Kerker), p. 151. Pergamon Press, Inc. New York.

Elsässer, H. 1963, The Zodiacal Light. Planet. Space Sci. 11, 1015.

Elsässer, H. 1970, The Zodiacal Light: Space Observations. Space Research X. (eds., T. M. Donahue, P. A. Smith, and L. Thomas), p. 244. North-Holland Pub. Co. Amsterdam.

Elsässer, H., and Schmidt, T. 1966, Rayleigh-Teilchen $\left(a<10^{-5} \mathrm{~cm}\right)$ im interplanetaren Raum? Z. Naturforsh. A 21, 1116.

Field, G. B., Partridge, R. B., and Sobel, H. 1967, Effects of Absorption Spectra of Ices on the Ultraviolet Extinction by Interstellar Grains. Interstellar Grains (ed., J. M. Greenberg and T. P. Roark), p. 207. NASA SP-140.

Giese, R. H. 1961, Streuung elektromagnetischer Wellen an absorbierenden und dielektrischen Kugelförmigen Einzelteilchen und an Gemischen solcher Teilchen. Z. Astrophys. 51, 119.

Giese, R. H. 1963, Light Scattering by Small Particles and Models of Interplanetary Matter Derived From the Zodiacal Light. Space Sci. Rev. 1, 589.

Giese, R. H. 1970, Model Computations Concerning Zodiacal Light Measurements by Space Missions. Paper presented at COSPAR, 13th meeting (Leningrad).

Giese, R. H., and Dziembowski, C. v. 1967, On Optical Models Approximating Observations of the Zodiacal Light Outside the Ecliptic. The Zodiacal Light and the Interplanetary Medium (ed., J. L. Weinberg), pp. 271-276. NASA SP-150.

Giese, R. H., and Dziembowski, C. v. 1969, Suggested Zodiacal Light Measurements From Space Probes. Planet. Space Sci. 17, 949.

Giese, R. H., and Siedentopf, H. 1962, Optische Eigenschaften von Modellen der interplanetaren Materie. Z. Astrophys. 54, 200.

Greenberg, J. M. 1970, Models of the Zodiacal Light. Space Research X (eds., T. M. Donahue, P. A. Smith, and L. Thomas), p. 225. North-Holland Pub. Co. Amsterdam.

Greenberg, J. M., Pedersen, N. E., and Pedersen, J. C. 1961, Microwave Analog to the Scattering by Nonspherical Particles. J. Appl. Phys. 32, 233.

Greenberg, J. M., Wang, R. T., and Bangs, L. 1971, Extinction by Rough Particles and the Use of Mie Theory. Nature (London) Phys. Sci. 230, 110-112.

Hanner, M. S. 1969, Light Scattering in Reflection Nebulae. Ph. D. Thesis. Rensselaer Polytechnic Institute.

Hanner, M. S. 1970, Zodiacal Light Models Based on Scattering by Silicate Particles. Paper presented at Amer. Astron. Soc. Meeting (Boulder, Colo.). 
Harwit, M. 1963, Infrared Appearance of Different Zodiacal Light Cloud Models. Paper presented at the 12th Int. Colloq. Astrophys. Inst. (Liege, Belgium).

Hemenway, C. L., and Hallgren, D. S. 1970, Time Variation of the Altitude Distribution of the Cosmic Dust Layer in the Upper Atmosphere. Space Research X (ed., T. M. Donahue, P. A. Smith, and L. Thomas), p. 272. North-Holland Pub. Co. Amsterdam.

Hemenway, C. L., Hallgren, D. S., and Coon, R. E. 1967, High Altitude Balloon-Top Collections of Cosmic Dust. Space Research VII (ed., R. L. Smith-Rose), p. 1423. North-Holland Pub. Co. Amsterdam.

Hemenway, C. L., Hallgren, D. S., Laudate, A. T., Patashnick, H., Renzema, T. S., and Griffith, O. K. 1970, A New High Altitude Balloon-Top Cosmic Dust Collection Technique. Paper presented at COSPAR, 13th meeting (Leningrad).

Holland, A. C. 1969, The Scattering of Polarized Light by Polydisperse Systems of Irregular Particles. NASA TN D-5458.

Holland, A. C., and Gagne, G. 1970, The Scattering of Polarized Light by Polydisperse Systems of Irregular Particles. Appl. Opt. 9, 1113.

Hulst, H. C., van de. 1947, Zodiacal Light in the Solar Corona. Astrophys. J. 105, 471.

Hulst, H. C., van de. 1957, Light Scattering by Small Particles. John Wiley \& Sons, Inc. New York.

Ingham, M. F. 1961, Observations of the Zodiacal Light From a Very High Altitude Station IV. The Nature and Distribution of the Interplanetary Dust. Mon. Notic. Roy. Astron. Soc. 122, 157.

Ingham, M. F., and Jameson, R. F. 1968, Observations of the Polarization of the Night Sky and a Model of the Zodiacal Cloud Normal to the Ecliptic Plane. Mon. Notic. Roy. Astron. Soc. 140, 473.

Jameson, R. F. 1970, Observations and a Model of the Zodiacal Light. Mon. Notic. Roy. Astron. Soc. 150, 207.

Kerker, M. 1969, The Scattering of Light and Other Electromagnetic Radiation. Academic Press, Inc. New York.

Lind, A. C. 1966, Resonance Electromagnetic Scattering by Finite Circular Cylinders. Ph. D. Thesis. Rensselaer Polytechnic Institute.

Little, S. J., O'Mara, B. J., and Aller, L. H. 1965, Light Scattering by Small Particles in the Zodiacal Cloud. Astron. J. 70, 346.

Peterson, A. W. 1963, Thermal Radiation From Interplanetary Dust. Astrophys. J. 138, 1218.

Peterson, A. W. 1964, Thermal Radiation From Interplanetary Dust II. Distribution of the Dust. Ann. N.Y. Acad. Sci. 119, 72.

Peterson, A. W., and MacQueen, R. M. 1967, Infrared Observations of Thermal Radiation From Interplanetary Dust at the Eclipse of November 12, 1966 (abstract). The Zodiacal Light and the Interplanetary Medium (ed., J. L. Weinberg), p. 89. NASA SP-150.

Powell, R. S., Woodson, P. E., III, Alexander, M. A., Circle, R. R., Konheim, A. G., Vogel, D. C., and McElfresh, T. W. 1967, Analysis of all Available Zodiacal Light Observations. The Zodiacal Light and the Interplanetary Medium (ed., J. L. Weinberg), pp. 225-241. NASA SP-150.

Richter, N. B. 1966, The Photometric Properties of Interplanetary Matter. Quart. J. Roy. Astron. Soc. 3, 179.

Singer, S. F., and Bandermann, L. W. 1967, Nature and Origin of Zodiacal Light. The Zodiacal Light and the Interplanetary Medium (ed., J. L. Weinberg), p. 379. NASA SP-150.

Smith, L. L., Roach, F. E., and Owen, R. W. 1965, The Absolute Photometry of the Zodiacal Light. Planet. Space Sci. 13, 207.

Stecher, T. P. 1965, Interstellar Extinction in the Ultraviolet. Astrophys. J. 142, 1683.

Taft, E. A., and Philipp, H. R. 1965, Optical Properties of Graphite. Phys. Rev. 138A, 197. 
Weinberg, J. L. 1964, The Zodiacal Light at 5300A. Ann. Astrophys. 27, 718.

Weinberg, J. L., 1970, Current Problems in the Zodiacal Light. Space Research X (eds., T. M. Donahue, P. A. Smith, and L. Thomas), p. 233. North-Holland Pub. Co. Amsterdam.

Weinberg, J. L., and Mann, H. M. 1968, Negative Polarization in the Zodiacal Light. Astrophys. J. 152, 665 .

\section{DISCUSSION}

BANDERMANN: Purely photometric, spectroscopic, polarimetric observations of the zodiacal light will not lead to definite answers about the physical properties of the particles. One must try to consider all the different types of evidences (zodiacal light as well as impact counts, deep sea sediments, etc.) and then look for collaboration toward an answer. 